\title{
Phytoprotection
}

\section{Distribution et détection du Sirococcus clavigignenti-juglandacearum au Québec}

\section{Innes et A. Rainville}

Volume 77, numéro 2, 1996

URI : https://id.erudit.org/iderudit/706102ar

DOI : https://doi.org/10.7202/706102ar

Aller au sommaire du numéro

Éditeur(s)

Société de protection des plantes du Québec (SPPQ)l

ISSN

0031-9511 (imprimé)

1710-1603 (numérique)

Découvrir la revue

Citer cet article

Innes, L. \& Rainville, A. (1996). Distribution et détection du Sirococcus clavigignenti-juglandacearum au Québec. Phytoprotection, 77(2), 75-78. https://doi.org/10.7202/706102ar
Résumé de l'article

La maladie du chancre du noyer cendré, causée par le Sirococcus clavigignenti-juglandacearum, affecte principalement le noyer cendré, Juglans cinerea, bien que le noyer noir, Juglans nigra, et les noyers hybrides puissent en être atteints. La maladie est présente dans plusieurs États américains ainsi qu'en Ontario et au Québec. Au Québec, elle a été rapportée pour la première fois en 1990, dans la région de Fort-Coulonge, en Outaouais. Depuis, de nouveaux foyers d'infection ont été notés en forêt naturelle ainsi que dans une plantation. En 1995, elle a été observée pour la première fois sur des semis de noyer dans deux pépinières forestières québécoises. Cette détection constitue le premier cas documenté de la maladie sur des semis de noyer cendré et de noyer noir dans des pépinières forestières. 


\title{
Communication brève / Short Communication \\ Distribution et détection du Sirococcus clavigignenti- juglandacearum au Québec
}

\author{
Louise Innes ${ }^{1}$ et André Rainville ${ }^{2}$
}

Reçu 1996-06-05; accepté 1996-09-02

\begin{abstract}
La maladie du chancre du noyer cendré, causée par le Sirococcus clavigignentijuglandacearum, affecte principalement le noyer cendré, Juglans cinerea, bien que le noyer noir, Juglans nigra, et les noyers hybrides puissent en être atteints. La maladie est présente dans plusieurs États américains ainsi qu'en Ontario et au Québec. Au Québec, elle a été rapportée pour la première fois en 1990, dans la région de Fort-Coulonge, en Outaouais. Depuis, de nouveaux foyers d'infection ont été notés en forêt naturelle ainsi que dans une plantation. En 1995, elle a été observée pour la première fois sur des semis de noyer dans deux pépinières forestières québécoises. Cette détection constitue le premier cas documenté de la maladie sur des semis de noyer cendré et de noyer noir dans des pépinières forestières.
\end{abstract}

Innes, L., and A. Rainville. 1996. Distribution and detection of Sirococcus clavigignenti-juglandacearum in Quebec. PHYTOPROTECTION 77 : 75-78.

Although the Sirococcus canker of butternut, caused by Sirococcus clavigignenti-juglandacearum, has caused damage only to butternut, Juglans cinerea, black walnut, Juglans nigra, and hybrids of walnut are also susceptible to a certain degree. The disease is present in the United States, in Ontario and in Quebec. In Quebec, the disease was first reported in 1990 near FortCoulonge, in the Outaouais region. Since then, new infection sites have been observed in natural stands and in one plantation. In 1995, this disease was isolated for the first time from seedlings in two forest nurseries in Quebec. This constitutes the first report of the disease on butternut and black walnut seedlings in forest nurseries.

Aux États-Unis, la maladie du chancre du noyer cendré (Sirococcus clavigignentijuglandacearum Nair, Kostichka \& Kuntz.) est très agressive (Tisserat et Kuntz 1984). Elle a complètement éliminé l'espèce dans les deux Carolines (Anderson et LaMadeleine 1978; Sinclair et al. 1987) et a presque entraîné sa disparition au Wisconsin (Kuntz et al. 1978; Tisserat et Kuntz 1984), au Minnesota, au Michigan et en Virginie (Ostry et al. 1994). Le noyer cendré (Juglans cinerea L.) y a d'ailleurs été désigné comme espèce forestière menacée de disparition par le service forestier du United States Department of Agriculture. La maladie a aussi été rapportée en Ontario (Davis et al. 1992), où elle semble s'être introduite à partir des États-Unis.

1. Ministère des Ressources naturelles du Québec, Direction de la conservation des forêts, Québec (Québec), Canada G1N 2C9

2. Ministère des Ressources naturelles du Québec, Direction de la recherche forestière, SainteFoy (Québec), Canada G1P 3W8 
Bien que le noyer cendré soit l'essence forestière la plus atteinte par la maladie, le noyer noir (Juglans nigra L.) et les noyers hybrides peuvent également être touchés (Orchard et al. 1982).

Le champignon pathogène cause un chancre fusiforme et pérenne localisé sur le tronc, les branches et les rameaux, et même, dans certains cas, sur les grosses racines exposées à l'air (Kuntz et al. 1978). Les chancres prendraient naissance dans des cicatrices foliaires, des bourgeons, des lenticelles, des blessures, des fentes naturelles sur l'écorce et, occasionnellement, dans des points où aucune blessure n'est apparente (Nair et al. 1979). Les jeunes chancres de couleur noirâtre continuent à se développer d'année en année et prennent la forme d'une cible elliptique très caractéristique. Éventuellement, les arbres couverts de plusieurs chancres meurent. Dans les sites où la maladie est bien installée, peu ou aucune regénération n'a lieu (Nair et al. 1979).

S. clavigignenti-juglandacearum est I'anamorphe du champignon classifié dans les Coelomycètes; I'hétéromorphe demeure inconnu. Peu de temps après l'infection, laquelle a généralement lieu au printemps, le champignon produit un stroma et des fructifications (pycnides) noirs sur l'écorce. Les hyphes du stroma se différencient en de petites colonnes de $0,5 \mathrm{~mm}$ de largeur et $1,5-2,0 \mathrm{~mm}$ de hauteur qui soulèvent et rompent la couche externe de l'écorce. Lors d'observations faites aux États-Unis, on a constaté que la contamination se fait par des conidies relâchées par les pycnides lors de périodes humides, du début du printemps jusqu'à l'automne (Sinclair et al. 1987). La sporulation à partir de chancres peut se continuer sur une période de 20 mois. La dissémination de la maladie semble se faire par le vent et les insectes. Les noix contaminées pourraient également transmettre la maladie aux jeunes arbres (Sinclair et al. 1987), mais ce mode de dissémination reste une hypothèse.

Dans le cadre du relevé général des insectes et des maladies des arbres du Québec, la maladie a été détectée pour la première fois en 1990, en forêt naturelle à Waltham (unité de gestion de la Coulonge; lat. $45^{\circ} 55^{\prime} \mathrm{N}$, long. $76^{\circ} 53^{\prime} \mathrm{O}$ ) et à Fort-Coulonge (unité de gestion de la
Coulonge; lat. $45^{\circ} 49^{\prime} \mathrm{N}$, long. $\left.76^{\circ} 41^{\prime} \mathrm{O}\right)$. Des prélèvements de branches affectees et de chancres au tronc ont été acheminés au laboratoire de pathologie forestière de la Direction de la conservation des forêts (DCF) du ministère des Ressources naturelles du Québec (MRN). En laboratoire, I'isolement de l'agent pathogène a permis d'identifier formellement le champignon comme étant le $S$. clavigignentijuglandacearum. Afin de confirmer cette identification, des prélèvements ont été envoyés à l'Institut de recherche biosystématique d'Ottawa. Des spécimens d'herbier ont été déposés dans la collection de champignons du laboratoire de pathologie forestière de la DCF et portent le numéro MRN 1943.

Puis en 1994, un projet de recherche visant à caractériser la variabilité génétique du noyer cendré ainsi qu'à effectuer la culture in vitro et la cryopréservation de lignées cellulaires a été initié conjointement par la Direction de la recherche forestière du MRN et le Service canadien des forêts. Dans le cadre de ce projet et du relevé annuel des insectes et des maladies, la maladie a été retrouvée en 1994, en forêt naturelle à Fassett (unité de gestion de la Basse-Lièvre; lat. $45^{\circ} 39^{\prime} \mathrm{N}$, long. $74^{\circ} 52^{\prime} \mathrm{O}$ ) et à Deux-Montagnes (unité de gestion de la Rivière-Rouge; lat. $45^{\circ} 40^{\prime} \mathrm{N}$, long. $74^{\circ} 11^{\prime} \mathrm{O}$ ) et dans une plantation à Sainte-Cécile de Milton (région de la Montérégie; lat. $45^{\circ} 29^{\prime} \mathrm{N}$, long. $72^{\circ} 48^{\prime} \mathrm{O}$ ). Puis en 1995 , elle a été relevée à Glen Sutton (région de la Montérégie; lat. $45^{\circ} 1^{\prime} \mathrm{N}$, long. $72^{\circ} 35^{\prime} \mathrm{O}$ ), à Frelighsburg (région de la Montérégie; lat. $45^{\circ} 1^{\prime} \mathrm{N}$, long. $72^{\circ} 50^{\prime} O$ ) et à Ascot Corner (unité de gestion de l'Estrie; lat. $45^{\circ} 27^{\prime} \mathrm{N}$, long. $\left.71^{\circ} 45^{\circ} \mathrm{O}\right)$. La figure 1 présente la distribution des points de détection de la maladie au sud du Québec.

En 1995, lors du contrôle phytosanitaire des plants en pépinières forestières, le $S$. clavigignenti-juglandacearum a été isolé, en culture, de plants d'un an de noyer cendré et de noyer noir présentant des symptômes de la maladie. Les pépinières étaient situées à Duchesnay (unité de gestion de Portneuf-Duchesnay; lat. $46^{\circ} 51^{\prime} \mathrm{N}$, long. $71^{\circ} 38^{\prime} \mathrm{O}$ ) et à Berthierville (unité de gestion de L'Assomption-Matawin; lat. $46^{\circ} 2^{\prime} \mathrm{N}$, long. $73^{\circ} 11^{\prime} \mathrm{O}$ ), loin de foyers connus d'infection. Ces cultures 


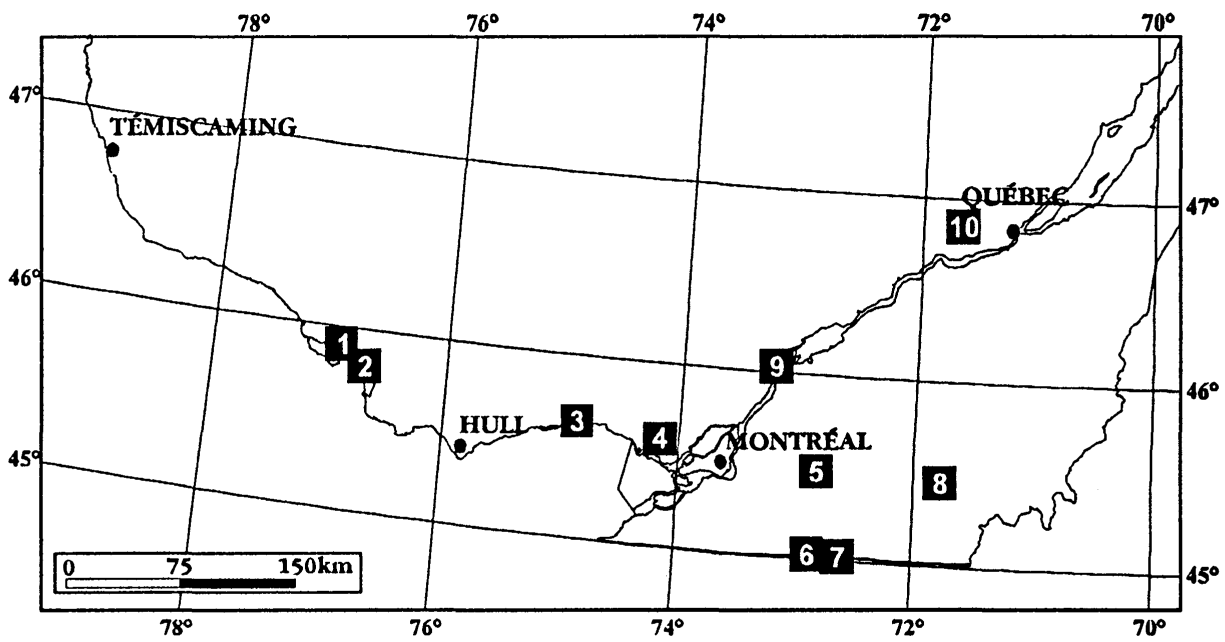

Figure 1. Distribution de $S$. clavigignenti-juglandacearum au Québec. Localisation des sites de récolte: Waltham 11, Fort-Coulonge 2 , Fassett 3, Deux-Montagnes 4 , Sainte-Cécile-deMilton 5 , Frelighsburg 6, Glen Sutton 7, Ascot Corner 8, Pépinière de Berthierville 9 , Pépinière de Duchesnay 10.

portent les numéros MRN 3-1-33 (Duchesnay) et MRN 3-1-41 (Berthierville). Ceci constitue la première mention de la présence de l'agent pathogène dans une pépinière forestière, sur le noyer cendré et sur le noyer noir. Les symptômes en pépinière se présentaient différemment de ceux rencontrés sur des arbres plus âgés en forêt naturelle. La porte d'entrée du champignon semblait être la cicatrice, à la base du plant, laissée lorsque la noix se détache. À cet endroit, une nécrose des tissus était bien évidente. Des fructifications de l'agent pathogène ont de plus été observées à la base de la tige, près du point d'attache de la noix.

La maladie du chancre du noyer cendré a été détectée pour la première fois au Québec en 1990. La maladie est très virulente et continue probablement à progresser. Depuis la première détection de la maladie, de nouveaux foyers d'infection s'ajoutent annuellement. Dans la province, le noyer cendré est une espèce indigène et il est plus que probable que l'épidémie ait atteint plusieurs des peuplements du sud du Québec. II est donc d'une importance capitale d'éviter de disséminer la maladie ailleurs au Québec, à partir de plants malades provenant de pépinières contaminées. De plus, un effort de détection de la maladie devra être envisagé afin de noter les nouveaux foyers d'infection et d'étudier l'épidémiologie de la maladie. Enfin, grâce à un projet de recherche mené par la Direction de la recherche forestière du MRN et le Service canadien des forêts, l'espèce a été plantée en dehors de son aire naturelle de distribution pour la mettre à l'abri d'une possible extinction comme ce fut le cas dans certains États américains. II permettra peut-être aussi d'identifier des arbres génétiquement résistants à la maladie afin de former des banques d'individus résistants.

\section{REMERCIEMENTS}

Les auteurs tiennent à remercier l'équipe de pathologie forestière et celle du contrôle phytosanitaire de la Direction de la conservation des forêts (DCF) du ministère des Ressources naturelles, particulièrement Guy Croteau, Chantal Lachance, Lucie Marchand et Solange Simard. Des remerciements s'adressent aussi à Gaston Laflamme du Service canadien des forêts et à Pierre Lortie de la Direction de la recherche forestière du MRN. 


\section{RÉFÉRENCES}

Anderson, R.L. et L.A. LaMadeleine. 1978. The distribution of butternut decline in eastern United States. USDA Forest Service, Forest Survey Report S-3-78. Northeastern Area State and Private Forestry, Broomall, Pennsylvania. 5 pp.

Davis, C.N., D.T. Myren et E.J. Czerwinski. 1992. First report of butternut canker in Ontario. Plant Dis. $76: 467$.

Kuntz, J.E., A.J. Prey, S. Jutte et V.M.G. Nair. 1978. The etiology, distribution, epidemiology, histology and impact of butternut canker in Wisconsin. Pages 69-72 in Walnut insects and diseases. USDA For. Serv., Gen. Tech. Rep. NC-52. North Central For. Exp. Stn. St. Paul, Minnesota.

Nair, V.M.G., C.J. Kosticka et J.E. Kuntz. 1979. Sirococcus clavigignenti-juglandacearum: an undescribed species causing canker on butternut. Mycologia $71: 641$.
Orchard, L.P., J.E. Kuntz et K.J. Kessler Jr. 1982. Reactions of Juglans species to butternut canker and implications for disease resistance. Pages 27-31 in Black walnut for the future. USDA For. Serv., Gen. Tech. Rep. NC-74. North Central For. Exp. Stn. St. Paul, Minnesota.

Ostry, M.E., M.E. Mielke et D.D. Skilling. 1994. Butternut- Strategies for managing a threatened tree. USDA For. Serv., Gen. Tech. Rep. NC-165. North Central For. Exp. Stn. St. Paul, Minnesota. 7 pp.

Sinclair, W.A., H.H. Lyon et W.T. Johnson. 1987. Diseases of trees and shrubs. Cornell University Press. Ithaca. 574 pp.

Tisserat, N. et J.E. Kuntz. 1984. Butternut canker: development on individual trees and increase within a plantation. Plant Dis. $68: 613-616$. 